\title{
Fibrillar morphologies of hydrogels obtained from a lamellar medium
}

\author{
Isabel E. Pacios, Carmen S. Renamayor ${ }^{* a}$ \\ CC. y TT. Fisicoquímicas, Facultad de Ciencias, UNED, Po Senda del Rey, 9, 28040 Madrid \\ Spain
}

\begin{abstract}
Hydrogels are suitable for multiple applications and their properties are strongly dependent on their morphology. Sponge-like morphologies are obtained in conventional hydrogels when the polymerization is performed in isotropic media. Fibrillar morphologies imparting new properties to hydrogels are expected when the reacting medium is anisotropic. Here, we synthesize such fibrillar hydrogels by polymerization in a lamellar medium formed by 1,4bis(2-ethylhexyl)sodium sulfosuccinate (AOT) and water. At high surfactant content this objective is achieved, and the thickness of the obtained fibrils $(0.1-1 \mu \mathrm{m})$ can be correlated with the physical properties of the lamellar system. At intermediate AOT concentrations, the morphology is hierarchical, with primary closed pores containing a secondary fibrillar structure that fills the pores. For low AOT concentrations, the crosslinking process is unhindered, and the hydrogels are mechanically consistent with a sponge-like morphology having large $\left(10-10^{2} \mu \mathrm{m}\right)$ void pores. The time evolution of the mesophase as polymerization advances is followed by small angle X-ray scattering.
\end{abstract}

Keywords: hydrogel, fibrillar morphology, lyotropic medium.

Published in European Polymer Journal, vol. 48, pp. 105-115 (2012)

\footnotetext{
${ }^{\text {a }}$ To whom correspondence should be addressed phone: $+34-913987386$, fax: $+34-913987390$, Email: csanchez@ccia.uned.es
} 


\section{Introduction}

Hydrogels are materials formed by hydrophilic polymers that are crosslinked into a network and have the capacity of absorbing large quantities of water. They are used in industry in important applications associated with technical (agricultural [1], electrical uses [2], electrophoretic matrices [3]) or biomedical areas (contact lens [4], drug delivery systems [5,6] or scaffolds used as cellular culture supports for tissue engineering [7]).

The design of these materials has been studied for many years. It is well known that molecular architecture and composition are relevant parameters for their properties, but other characteristics as the mechanical strength, the ease of handling or the surface/volume ratio are also important. Recently a great deal of attention is being paid to porosity. The control of the type (open $v s$. closed) and the size of pores (macro, meso or nanopores) extend and improve the hydrogels applicability [5,8]. For example, connected pores of micrometer size (20-200 $\mu \mathrm{m})$ and fibrillar networks are adequate materials for scaffolds in tissue engineering, facilitating cell seeding and providing effective transport of nutriments to growing cells $[9,10]$. Fibers within a polymer scaffold contribute to tensile strength, and alignment of fibers is useful for creating anisotropic materials properties. Fibers with diameters of similar scale to those of the natural structures would enable the provision of tailored materials for tissue engineering, e. g. fiber diameters equal to $0.5 \mu \mathrm{m}$ improves all the fundamental biological events of nerve regeneration in comparison with nanofibrous scaffolds [11].

There are many works on fibrillar networks obtained by self-assembling [12]. Nevertheless, crosslinked hydrogels with fibrillar structure have been rarely obtained. Among the different methods to obtain porous hydrogels (e.g. porogen leaching or gas bubbling), an interesting way to control the morphology is the template synthesis. Lyotropic liquid crystals (LLC) can be employed as structure directing agents $[13,14]$. Usually, the replication of the original LLC structure is not obtained [15], and the gels present morphologies whose features appear on length scales larger than those of the starting phases [16]. In spite of this, a new morphology usually appears which is different from that formed in LLC-free water solutions, as a consequence of the polymerization-induced phase separation within the lyotropic medium. The final morphology is the result of a balance between the changes produced in the phase diagram due to polymerization, the kinetics of the reaction, and the diffusion process $[17,18]$. Previous studies indicate that the morphology varies with the molecular structure of the monomer and also with the ordered structure of the mesophase [19,20]. It has been also 
demonstrated that the polymerization kinetics depends on the LLC organization [21] and therefore affects to the final polymer structure [22].

Earlier we have studied the crosslinking polymerization of poly(N,N-dimethylacrylamide), PDMAA, in isotropic surfactant/water mixtures that become anisotropic with polymerization $[23,24]$. In these conditions hydrogels with sponge-like morphologies are obtained and the formation of the ordered domains induces the following porogen effects: (i) more macropores with a better defined geometry are formed when the developed mesophase is hexagonal. (ii) smaller pores are generated from lamellar mesophases.

The overall aim of this work is the obtention of fibrillar hydrogels with controlled morphology. With this purpose, we have synthesized hydrogels in media having anisotropic organization in the initial mixture. We are inspired by the fact that when polymers are obtained in thermotropic liquid crystals they render oriented fibrillar networks $[25,26]$. Here, the samples have lamellar order at the beginning in contrast with a previous work where the initial system was isotropic. The surfactant concentration is varied widely, within the range where the mesophase is lamellar (keeping the comonomers concentration constant), in order to make a systematic study analyzing the evolution of the LLC organization that is induced by the polymerization process. The final morphology of the hydrogels is characterized in an attempt to correlate morphology with mesophase structure and polymerization steps. With this purpose, two polymerization methods have been used: (i) a traditional thermal initiation process, which allow obtaining sufficient amount of sample enough to characterize the morphology, glass transition, swelling, and pore diameter of hydrogels; (ii) a polymerization initiated with X-rays, which allow us to follow the development in situ, of the ordered mesophase with time, by SAXS.

\section{Materials and Methods}

\subsection{Materials}

1,4-bis(2-ethylhexyl)sodium sulfosuccinate (AOT) and $N, N$-dimethylacrylamide (DMAA) (99\% purity) were from Sigma and Aldrich, respectively. $N, N^{\prime}$-methylenebis(acrylamide) (BA) (99.5\% purity) was from Fluka. AIBN ( $\alpha, \alpha^{\prime}$-azobis(isobutironitrile) from Fluka, was crystallized from methanol. AOT was freeze-dried before use and the other reagents were used as received. Deionized water (Milli-Q) was used for the preparation of samples. 


\subsection{Sample preparation}

Two set of samples (named $E_{P}$ and $E_{0}$ ) were prepared having the same comonomer composition (3.5 wt\% N,N-dimethylacrylamide, $0.39 \mathrm{wt} \% \mathrm{~N}, \mathrm{~N}$-methylenebis(acrylamide)) and a fraction of 1,4-bis(2-ethylhexyl)sodium sulfosuccinate ranging from 0 to $71 \mathrm{wt} \%$. The samples corresponding to the set $E_{P}$ have also an initiator ( $\alpha, \alpha^{\prime}$-azobis(isobutironitrile)) at concentration $0.045 \mathrm{wt} \%$. Samples are named as $\# \mathrm{E}_{\mathrm{P}}$ or $\# \mathrm{E}_{0}$ where $\#$ refers to the concentration of 1,4-bis(2-ethylhexyl)sodium sulfosuccinate expressed in percentage by weight. In the following, the term \#E is used when we want to refer indistinctly to samples of both sets. Table 1 contains the 1,4-bis(2-ethylhexyl)sodium sulfosuccinate content in the samples studied here.

To obtain these samples, the following procedure was used: For samples $\mathrm{E}_{0}$, a stock solution was prepared containing the comonomers and a part of the water. For the set $E_{P}$, a similar stock solution was prepared, also containing the initiator. The final composition of each sample was reached by weighing the same amount of the stock solution, and the appropriate amounts of 1,4-bis(2-ethylhexyl)sodium sulfosuccinate and water. Samples were homogenized mixing back and forth for several days. After this process they were allowed to equilibrate at $25^{\circ} \mathrm{C}$.

Table 1 1,4-bis(2-ethylhexyl)sodium sulfosuccinate concentration (AOT) of the samples

\begin{tabular}{llll}
\hline Sample & $\begin{array}{l}\text { AOT } \\
(\mathrm{wt} \%)\end{array}$ & Sample & $\begin{array}{l}\text { AOT } \\
(\mathrm{wt} \%)\end{array}$ \\
\hline $0 \mathrm{E}$ & 0 & $43 \mathrm{E}$ & 42.9 \\
$7 \mathrm{E}$ & 7.1 & $46 \mathrm{E}$ & 45.9 \\
$14 \mathrm{E}$ & 14.3 & $50 \mathrm{E}$ & 49.9 \\
$21 \mathrm{E}$ & 21.4 & $57 \mathrm{E}$ & 57.1 \\
$29 \mathrm{E}$ & 28.6 & $64 \mathrm{E}$ & 64.2 \\
$36 \mathrm{E}$ & 35.7 & $71 \mathrm{E}$ & 71.4 \\
\hline
\end{tabular}

Samples corresponding to set $\mathrm{E}_{0}$ were characterized by optical microscopy and SAXS in order to determine the structure of the system before polymerization. The X-ray induced polymerization was also monitored and analyzed in these samples.

Samples $E_{P}$ were characterized before polymerization by optical microscopy. Then, they were 
introduced in a thermostatic bath where the thermal induced polymerization was carried out at $65{ }^{\circ} \mathrm{C}$ for 24 hours. After polymerization, the upper zone of the samples was disregarded to eliminate the interfacial effects between the sample and the air. Then, a fraction of the samples was separated for characterizing the system by SAXS and optical microscopy. The remainder was treated to isolate the polymer and characterize it by DSC, SEM and swelling measurements. With this purpose, the samples were taken out of the moulds, cut into small pieces and immersed in deionized water. The hydrogels mechanically consistent were washed with water that was frequently changed, and the conductivity was checked to assure that the 1,4-bis(2-ethylhexyl)sodium sulfosuccinate was eliminated. The hydrogels without mechanical consistency were dispersed in water and precipitated over acetone three times. The purification was controlled with ${ }^{1} \mathrm{H}$ NMR by checking the absence of peaks corresponding to 1,4-bis(2-ethylhexyl)sodium sulfosuccinate. After eliminating the 1,4-bis(2ethylhexyl)sodium sulfosuccinate, the hydrogels were swollen in deionized water to equilibrium and freeze-dried to obtain xerogel specimens.

\subsection{Techniques}

Microscopy. A microscope (Nikon Labophot-2) equipped with a Nikon camera (model DS$5 \mathrm{M})$ and crossed polarizers was employed to determine the anisotropy of the samples. During these measurements, the samples were placed between a glass slide and a cover slip.

Small Angle X-ray Scattering (SAXS). The experiments were performed at the European Synchrotron Radiation Facility (ESRF) in Grenoble on the beam line BM16. The samples were irradiated with a wavelength $\lambda=0.979 \AA$, and two-dimensional images were recorded using a CCD detector (MARCCD 165) with a resolution of $1024 \times 1024$ pixels, and a pixel size of $159 \mu \mathrm{m}$. The sample-detector distance was $1016 \mathrm{~mm}$ with the flat holder, and 1020 $\mathrm{mm}$ with the capillary holder. Images were integrated to obtain the scattered intensity as a function of the modulus of the scattering vector, $q=(4 \pi / \lambda) \sin \theta$, where $2 \theta$ is the scattering angle, and were normalized to compensate for the intensity fluctuations of the synchrotron source. Each sample was measured three times in three different locations to average the position of the peaks, in order to obtain the error bar that will appear on the results.

Polymerization induced by X-ray irradiation was followed with time in the range $0.5-60 \mathrm{~s}$, obtaining $120 \mathrm{SAXS}$ diffractograms with irradiation times of $0.5 \mathrm{~s}$ each. The samples were not irradiated longer in order to prevent degradation. 
Differential Scanning Calorimetry (DSC). The equipment was a Mettler Toledo calorimeter provided with a DSC822 oven and a sub-ambient cooling unit. Specimens of dried hydrogel of about 4-5 mg, determined within $0.02 \mathrm{mg}$ by means of a Mettler Toledo AG285 electrobalance, were employed. Two runs were performed with each specimen, in a nitrogen atmosphere, using an empty aluminium cell as a reference. Samples were heated at $220^{\circ} \mathrm{C}$ for 10 min (a temperature well below the beginning of thermal instability of PDMAA xerogels). A first run was then scanned at $20^{\circ} \mathrm{C} \min ^{-1}$ in the range $220-60{ }^{\circ} \mathrm{C}$, and a second run was scanned at $20{ }^{\circ} \mathrm{C} \mathrm{min}{ }^{-1}$ from 60 to $220{ }^{\circ} \mathrm{C}$. Only second run results are used for calculation. Among the several criteria proposed for the determination of $T_{g}$, we have taken the inflection point provided by the software of the instrument.

Swelling. After removing the AOT, pieces of the consistent swelled gels were used to determine the swelling gravimetrically. Each sample was weighed before and after freeze drying and the swelling $(S)$ was obtained as:

$$
S=\frac{m_{s}-m_{d}}{m_{d}}
$$

where $m_{s}$ is the mass of hydrogel swollen to equilibrium and $m_{d}$ is the mass of dry gel.

Scanning Electron Microscopy (SEM): The measurements were carried out in a JEOL JSM 6400 or in a Hitachi S-3000N electron microscope. The inner parts of the xerogel specimens were revealed by simply cutting them. They were coated with gold or gold-palladium by means of a Balzers SCD 004 or a Polaron Range SC7620 sputter-coater. Several specimens of each sample were studied, and a number of micrographs of any relevant portion were taken for each specimen.

Image processing. SEM micrographs were analysed with the ImageJ software [27]. After calibrating with a known scale, the program measures the Feret's diameter of each dark region, which in the micrographs corresponds to a pore of the hydrogels. The Feret's diameter represents the longest distance between any two points along the selection boundary (dark region). The program has also been used to obtain a statistical average of the thickness of the fibrillar structures.

\section{Results and discussion}

\subsection{Before polymerization}


The binary system (AOT/water) presents a micellar region at concentrations under $5 \mathrm{wt} \%$, a biphasic region formed by a micellar phase and a lamellar phase between 5 and $15 \mathrm{wt} \%$, a lamellar monophasic region from about 15 to $72 \mathrm{wt} \%$ and at higher AOT concentrations cubic and hexagonal mesophases are formed [28]. In the system studied here, samples with $7 \mathrm{wt} \%$ and $14 \mathrm{wt} \%$ of AOT (7E and 14E) before polymerization are macroscopically biphasic; the upper phase is transparent and the bottom one is turbid. At room temperature, the bottom phase of sample $7 \mathrm{E}$ is $30 \%$ of the total volume, while for sample $14 \mathrm{E}$ is $78 \%$ of the total volume. The other samples are transparent, viscous fluids, and macroscopically monophasic. Optical micrographs obtained with crossed polarizers indicate that the monophasic samples present optical anisotropy, although for the biphasic samples only the bottom phase shows optical anisotropy. Fig. 1 depicts the micrographs with crossed polarizers for some selected samples, showing the typical marbled texture of a lamellar phase. Sample 46E exhibits a low birefringence, and the same behaviour has been observed for sample 50E. This low birefringence could correspond to a change in the optical sign of the lamellar phase, as it has been reported for the AOT/water system at similar concentration [29]. This behaviour is important because, not only the optical sign, but other physical properties change at this point, as we will see below. The micrographs corresponding to higher AOT concentrations show clear focal conic domains, which could be indicative of a change in the elastic constants of the layers [30].

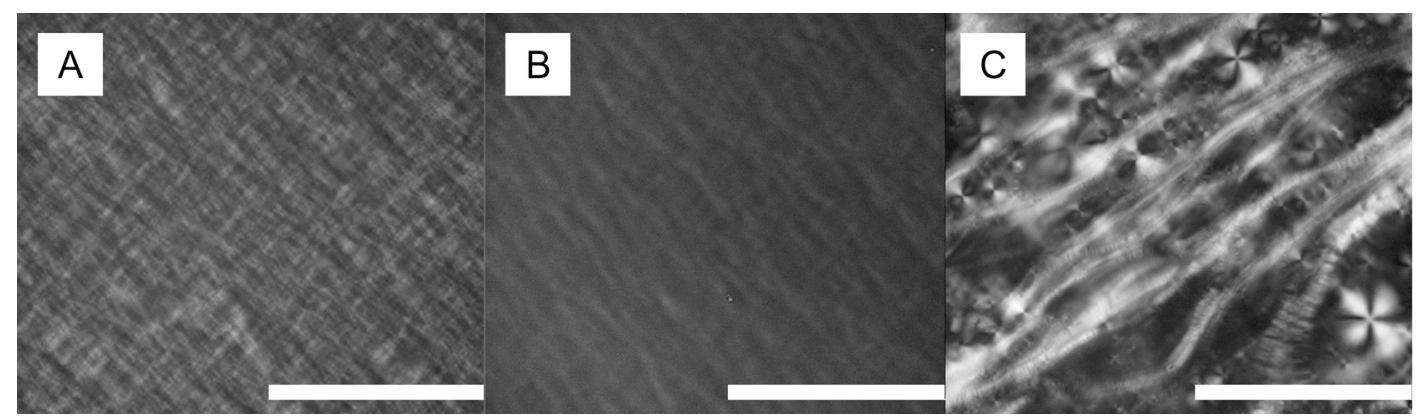

Fig. 1 Micrographs with crossed polarizers at room temperature before polymerization corresponding to samples: A) $\left.29 \mathrm{E}_{\mathrm{P}}, \mathrm{B}\right) 46 \mathrm{E}_{\mathrm{P}}$ and C) $57 \mathrm{E}_{\mathrm{P}}(\mathrm{bar}=100 \mu \mathrm{m})$.

The structural order of samples before polymerization has been determined for the set $\mathrm{E}_{0}$. In accordance with the optical microscopy, SAXS diffractograms show, for monophasic samples and the bottom phase of the biphasic samples, two or three peaks at relative positions of the scattering vectors 1:2:3, which agree with a lamellar structure (see the first diffractogram in 
each of Fig. 2 andFig. 3). On the other hand, the upper phase shows a broad band between 1 and $5 \mathrm{~nm}^{-1}$ characteristics of an isotropic phase, and a peak with very low intensity (at around $0.6 \mathrm{~nm}^{-1}$ ) that could be produced by a small contamination with the bottom phase.

The lamellar phase is formed by arrays of surfactant bilayers swollen by water. The thickness of the AOT bilayer $\left(d_{0}\right)$ remains constant and the water layer distance $\left(d_{w}\right)$ depends on the water content. The long period, $d$, is $d=d_{0}+d_{w}$ and can be calculated from the position of the first order Bragg peaks of the lamellar phase as $d=2 \pi / q$, where $q$ is the scattering vector corresponding to the first diffraction peak. In the AOT/water system the bilayer thickness is related with the composition by the following dilution law [31]:

$$
d_{0}=\phi_{A O T} d
$$

where $\phi_{A O T}$ is the AOT volume fraction. In the system studied here, there is also water soluble comonomers (BA and DMAA) and, as a first approximation, it is possible to assume that they are located in the water layer, and, in such a case, the equation (2) should also be valid with $\phi_{A O T}$ being the AOT volume fraction in the whole mixture. Under this assumption, the bilayer thickness can be calculated for monophasic samples, obtaining $d_{0}=1.88 \pm 0.05 \mathrm{~nm}$, which is slightly lower than that reported ${ }^{31}$ for the AOT/water system $\left(d_{0}=1.96 \mathrm{~nm}\right)$. Two possible explanations can be argued (i) An interaction between the comonomers and the polar AOT head group. This effect was previously reported for similar systems [32,33]. (ii) a modification of the effective dielectric permittivity of the solvent produced by the addition of the monomer. The comonomers are organic compounds with low relative dielectric permittivity whereas for water $\varepsilon_{\mathrm{r}} \approx 80$. Therefore, the addition of a small amount of these comonomers will diminish the permittivity of the medium, with the consequent increase of the electrostatic repulsion between the head groups, as was previously reported [34].

Once the bilayer thickness is known, equation (2) allows determination of the AOT volume fraction of the lamellar phase in the biphasic samples, obtaining 0.168 and 0.174 for samples $7 \mathrm{E}_{0}$ and $14 \mathrm{E}_{0}$, respectively. These values are higher than the global AOT content $\left(\phi_{A O T}=\right.$ 0.065 and 0.131 , respectively) as expected, since there is a macroscopic phase separation.

\subsection{Polymer segregation induced and monitored by $\mathrm{X}$-ray irradiation}

It is expected that, not only the initial organization of the system, but also the polymerization process have to play an important role in the gel morphology. Therefore, it seems interesting to follow the evolution of the system's structure during polymerization. 
It has been previously reported that $N, N$-dimethylacrylamide is able to polymerize by irradiation either with $\gamma$ or X-rays $[35,23]$. Samples corresponding to set $E_{0}$ were polymerized with X-ray irradiation, and the process was followed in situ by the change in the scattering curves; from the initial homogeneous mixture (comonomers, AOT and water) to the polymerized state (hydrogel, AOT, water and residual monomers).

Before polymerization, monophasic samples are transparent and after a few seconds of irradiation time, the samples become turbid in the region of the capillary where the X-rays traverse the sample, due to the phase separation process produced by the polymerization.

As was detailed above, samples $7 \mathrm{E}_{0}$ and $14 \mathrm{E}_{0}$ are macroscopically biphasic, and both phases were studied separately. The upper phase remains isotropic with polymerization. In the bottom phase, which is lamellar from the beginning, the peak position moves to lower $q$ values when polymerization progresses, indicating an increase of $d$ or swelling of the lamellae, that would be explained by considering two scenarios:

(i) The polymer is partially included in the lamellar phase. This behaviour has been observed previously for linear polymers, when the polymer coils are small enough to penetrate into the aqueous layer [36], therefore it is not expected for the crosslinked polymers studied here.

(ii) The polymer is excluded from the lamellae and draws with it part of the water and also some AOT, due to an interaction between AOT and the crosslinker (BA) [23] which carries non-substituted hydrogens and, as a result, is able to form H-bonds with the polar head group of the surfactant. Thus, the bottom phase is formed by two microphases that do not separate macroscopically: an isotropic microphase which is composed of the whole polymer, water, and a part of the AOT; and a surfactant rich microphase composed of the remaining AOT and water arranged in ordered domains. Consequently, the lamellar spacing can increase or decrease depending on the distribution of the AOT and water in these two microphases.

The diffractograms corresponding to monophasic samples $\left(21 \mathrm{E}_{0}-64 \mathrm{E}_{0}\right)$ show, before polymerization, two peaks at relative position 1:2, characteristic of the lamellar order (in samples $21 \mathrm{E}_{0}$ to $57 \mathrm{E}_{0}$ the third diffraction peak can also be observed). At the initial stage of polymerization, there is not an appreciable change in the SAXS profiles, but, after 6 seconds of irradiation the intensity of these peaks diminishes without changing their positions. Additionally, two new peaks appear, and their relative positions are also in accord with a lamellar structure. Since they have a position differing from that of the previous peaks, their 
appearance reveals that a new lamellar phase is developing. As the polymerization progresses, the new peaks move to higher $q$ values and their intensity increases, indicating that the new lamellar phase shrinks as the polymer is formed, while the structure of the initial lamellar phase remains invariable. This constancy suggests that the system is highly constricted around the polymerized regions due to the high viscosity and the diffusion effects are greatly hindered. As an example, Fig. 2 depicts the behaviour of sample $46 \mathrm{E}_{0}$.

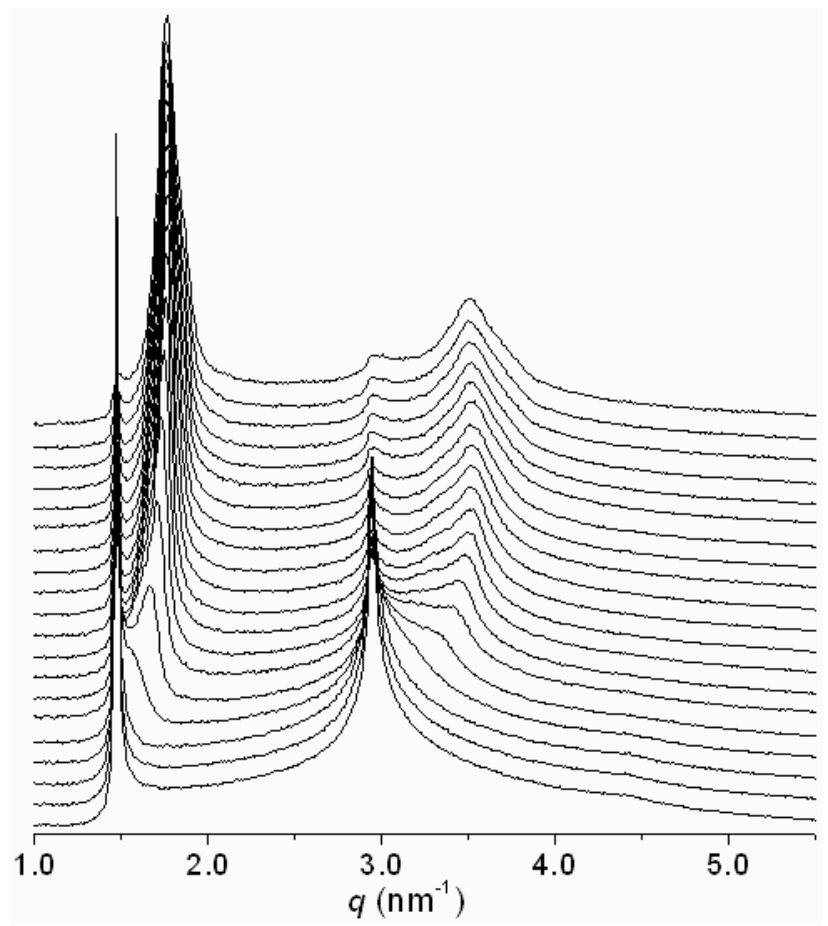

Fig. 2 X-ray induced polymerization of sample $46 \mathrm{E}_{0}$. Selected SAXS diffractograms for cumulative irradiation times in the range $0.5-60 \mathrm{~s}$, starting at the bottom of the graph. SAXS profiles have been progressively shifted upwards for better visualization. Intensity in arbitrary units.

The evolution of sample $71 \mathrm{E}_{0}$ with $\mathrm{X}$-ray irradiation is different. At the beginning, the pattern shows two peaks, whose positions agree with a lamellar order (Fig. 3). When polymerization starts there is no change in the diffractograms, but after nine seconds of irradiation, the intensity of these peaks diminishes without changing their positions and a small shoulder appears in both peaks at higher $q$ vector values. Until this moment it is the same behaviour that was observed for samples $21 \mathrm{E}_{0}$ to $64 \mathrm{E}_{0}$, indicating that the polymerization shrinks the lamellar domains close to the growing polymer chains. Nevertheless, after $12.5 \mathrm{~s}$ of cumulative irradiation time nine peaks can be observed. Their relative positions agree with the 
coexistence of two anisotropic phases, one lamellar (with two peaks at relative positions 1:2) and another bicontinuous cubic (Ia $\overline{3} \mathrm{~d}$ ) characterized by the following ratios for its reflection positions: $6^{1 / 2}: 8^{1 / 2}: 14^{1 / 2}: 16^{1 / 2}: 20^{1 / 2}: 22^{1 / 2}: 24^{1 / 2}$, where the last peak overlaps with the second order diffraction of the lamellar phase. In all the experiments it can be observed that the initial lamellar structure does not disappear, which would indicate that the polymerization yield is lower than $100 \%$. In spite of this, the samples cannot be irradiated longer time in order to prevent degradation.

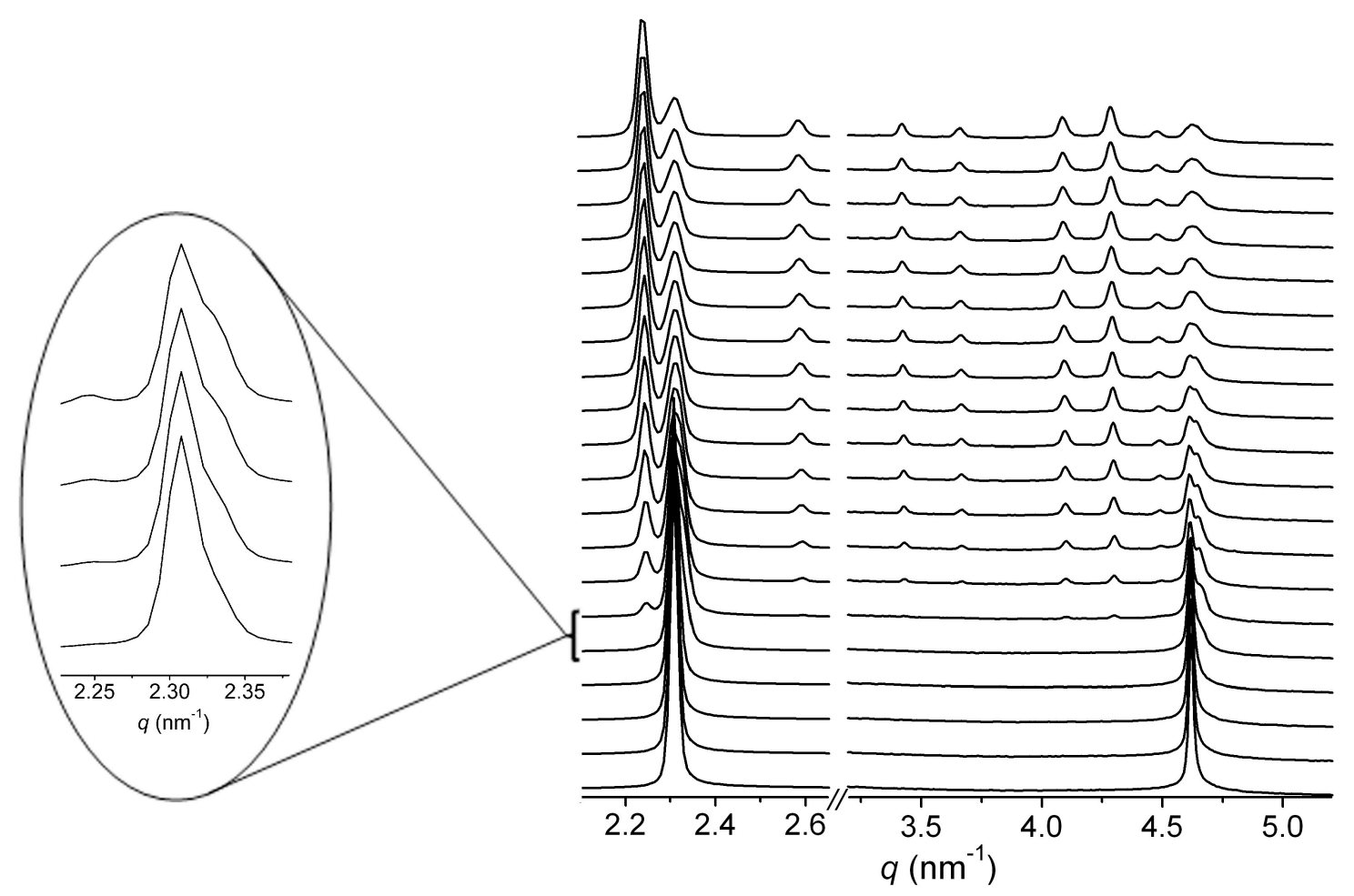

Fig. 3 X-ray induced polymerization of sample $71 \mathrm{E}_{0}$. Selected SAXS diffractograms for cumulative irradiation times in the range $0.5-60 \mathrm{~s}$ (starting at the bottom of the graph). SAXS profiles have been progressively shifted upwards for better visualization. The insert is an amplification of the region $2.25-2.35 \mathrm{~nm}^{-1}$ showing the appearance of a shoulder in the first order diffraction of the lamellar phase. Intensity in arbitrary units.

\subsection{Polymerized samples}

After polymerization, sample $7 \mathrm{E}_{\mathrm{P}}$ has three macroscopic phases, while samples $14 \mathrm{E}_{\mathrm{P}}$ and $21 \mathrm{E}_{\mathrm{P}}$ are biphasic. The bottom phase is turbid and its proportion increases with the global AOT content. The central region of sample $7 \mathrm{E}_{\mathrm{P}}$ would correspond to a diffusion zone between the upper and the bottom phases. Sample $21 \mathrm{E}_{\mathrm{P}}$ was monophasic before polymerization but its 
composition is close to the region where two phases appear because the presence of DMAA induces instability of the lamellar phase, so that the curves of coexistence become progressively displaced toward higher AOT concentrations as DMAA is being added [37]. For this reason, when temperature increases to $65^{\circ} \mathrm{C}$ two macroscopic phases are formed and, when the temperature decreases after polymerization, these phases remain due to the high viscosity of the system. The other samples, with higher AOT concentration, are macroscopically monophasic and turbid.

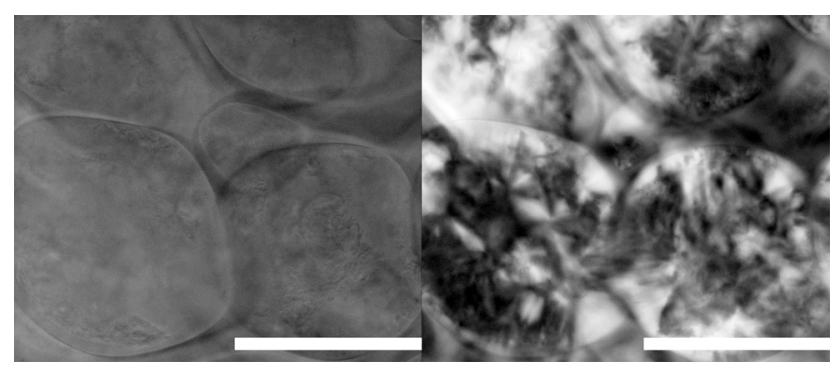

Fig. 4 Micrographs corresponding to the upper phase of sample $14 \mathrm{E}_{\mathrm{P}}$, without (left) and with (right) crossed polarizers. (bar $=100 \mu \mathrm{m})$.

Micrographs obtained with crossed polarizers show that, in sample $7 \mathrm{E}_{\mathrm{P}}$ the central and the bottom phases present a marbled lamellar texture while in its upper phase no texture has been noticed. The other samples present a marble texture, characteristic of the lamellar texture. As

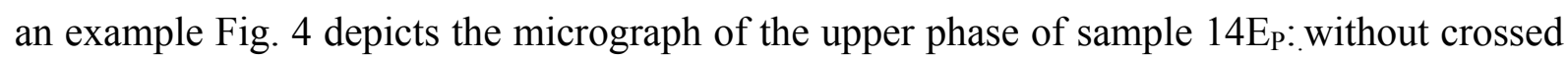
polarizers (left) a macroporous structure can be observed, while the micrograph obtained with crossed polarizers (right) reveals that these pores are filled by anisotropic domains.

Table 2 Lamellar spacing corresponding to the macroscopic phases (upper and bottom) of the samples with lower AOT content after polymerization.

\begin{tabular}{|c|c|c|c|c|}
\hline \multirow[b]{2}{*}{ Sample } & \multicolumn{2}{|c|}{ AIBN } & \multicolumn{2}{|c|}{ X-ray } \\
\hline & $\begin{array}{l}\text { Upper } \\
d(\mathrm{~nm})\end{array}$ & $\begin{array}{l}\text { Bottom } \\
d(\mathrm{~nm})\end{array}$ & $\begin{array}{l}\text { Upper } \\
d(\mathrm{~nm})\end{array}$ & $\begin{array}{l}\text { Bottom } \\
d(\mathrm{~nm})\end{array}$ \\
\hline $7 E$ & $15 \pm 1 \quad 13 \pm 1^{a}$ & $-b$ & - & $12.6 \pm 0.1$ \\
\hline $14 \mathrm{E}$ & $8.8 \pm 0.2$ & $8.63 \pm 0.02$ & - & $11.0 \pm 0.1$ \\
\hline $21 \mathrm{E}$ & $7.36 \pm 0.06$ & $7.05 \pm 0.05$ & $8.7 \pm 0.1$ & \\
\hline
\end{tabular}

\footnotetext{
${ }^{b}$ The amount of sample in this region was not enough to allow the SAXS characterization.
} 
It is also interesting to analyse the structure of samples $E_{0}$ and $E_{P}$ after polymerization, in order to compare both methods of synthesis. The lamellar spacing of the samples with macroscopic phase separation (7E, 14E, 21E) obtained with both polymerization methods can be compared from data presented in Table 2. The differences can be due to: (i) the polymerization process (initiator $v s \mathrm{X}$-ray irradiation) and (ii) the polymerization temperature ( $65{ }^{\circ} \mathrm{C}$ for AIBN polymerization and room temperature for X-ray induced polymerization). In fact, the composition of the phases in biphasic samples varies with temperature and therefore the initial state should also be different. On the other hand, the structure of monophasic samples (29E to 64E) is similar after polymerization (Fig. 5). The scattering curves of these agree with a lamellar structure and a SAXS pattern characteristic of the AOT/water system [38]: (i) they show a broad hump for wave vectors between 1 and $5 \mathrm{~nm}^{-1}$; and (ii) for some compositions the second order Bragg peak has higher intensity than the first order diffraction peak.

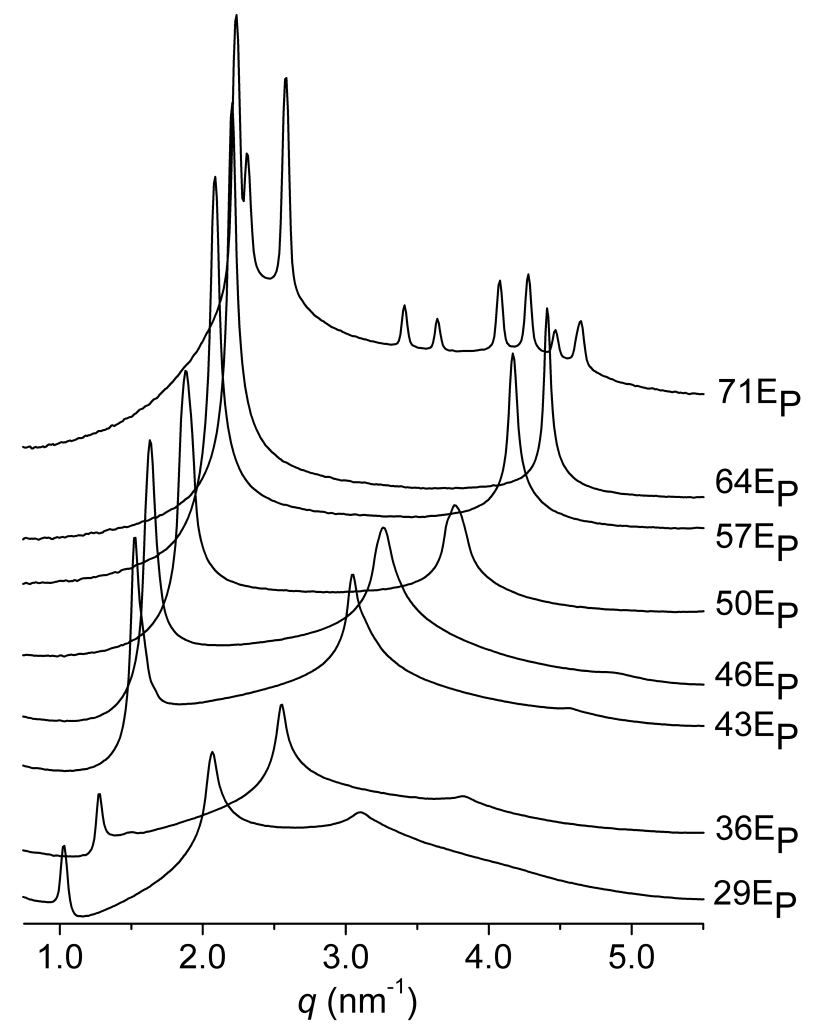

Fig. 5 SAXS diffractograms of macroscopic monophasic samples corresponding to samples polymerized with AIBN $\left(E_{P}\right)$. SAXS profiles have been progressively shifted upwards for better visualization. Intensity in logarithmic scale (arbitrary units).

Nevertheless, in the diffractograms of the $E_{P}$ samples there is no experimental evidence of the initial structure after polymerization (Fig. 5), while, as it was explained before, for the $\mathrm{E}_{0}$ 
samples the peaks corresponding to the initial structure do not completely disappear (Fig. 2). The structure of samples $\mathrm{E}_{0}$ can be understood taking into account that when polymer chains grow the polymer retains part of the water and, consequently, the local AOT/water concentration increases around the chains. Therefore, the SAXS profiles show two groups of signals: one corresponding to the initial system and other characteristic of the concentrated AOT regions around the chains. When the number of chains increases, more concentrated AOT regions are formed. Nevertheless, its structure is almost constant because the viscosity of the system is high enough to prevent the diffusion of the water.

Fig. 6 depicts that the long period that appear after polymerization decreases upon increasing the global AOT volume fraction, $\phi_{A O T}$ (calculated with the following densities: $\rho_{A O T}=1.1$ $\mathrm{g} / \mathrm{cm}^{3}$ and $\left.\rho_{P D M A A}=1.06 \mathrm{~g} / \mathrm{cm}^{3}\right)$. The similarity of these long period values for both groups of samples supports the idea that the diffusion is slow.

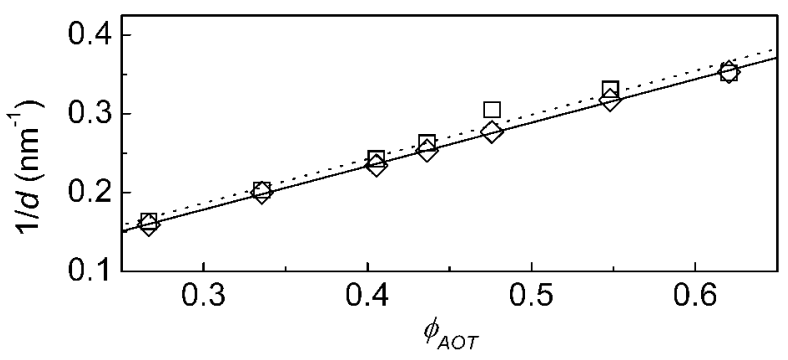

Fig. 6 Inverse of the long period $(1 / d)$ after polymerization as a function of global AOT volume fraction, $\phi_{\text {АОт }}$. Samples $E_{\mathrm{P}}$ : squares and dotted line; samples $\mathrm{E}_{0}$ : diamonds and solid line.

It was previously reported that polymerization induces a phase separation between an isotropic polymer-rich microphase and a surfactant-rich microphase (ordered domains). According to this, the following expression was previously developed [23]:

$$
\frac{1}{d}=\left(\frac{1}{d_{0}}\right)\left[(1-c) \phi_{A O T}+K \phi_{p o l}\right]
$$

Where, $\phi_{\text {pol }}$ is the global polymer volume fraction; $K$ is a constant characteristic of the system $(1.8 \pm 0.1$ for this polymer [37]) and $c$ is the AOT partition constant due to the interaction between the crosslinker (BA) and the AOT, defined as the volume fraction of AOT in the isotropic phase with respect to the global AOT in the sample. Here, $c$ can be disregarded 
because the fraction of BA is low and, consequently, it can be expected a low concentration of AOT in the polymer rich phase. Under this assumption, Fig. 6 should render $d_{0}$ and $\phi_{\text {pol }}$ from the slope and the intercept, respectively. If the local yield of polymerization were $100 \%$, $\phi_{\mathrm{pol}}=0.038$, and the surfactant rich phase would be formed by the whole surfactant and part of the water, therefore $d_{0}$ would be $1.96 \mathrm{~nm}$. After fitting the experimental data, values of $\phi_{\mathrm{pol}}=0.02 \pm 0.01$ and $d_{0}=1.82 \pm 0.07 \mathrm{~nm}$ are obtained for samples $\mathrm{E}_{\mathrm{P}}$, whereas for samples $\mathrm{E}_{0}$, values of $\phi_{\text {pol }}=0.012 \pm 0.004$ and $d_{0}=1.81 \pm 0.02 \mathrm{~nm}$ are obtained. Consequently, the results obtained suggest a low polymerization yield (about 53\% for samples $E_{P}$ and 32\% for samples $\mathrm{E}_{0}$ ). As was explained above, the presence of residual monomer in the lamellar structure induces a decrease of the bilayer thickness that would explain the low value of $d_{0}$ as compared with the expected value $(1.96 \mathrm{~nm})$.

Nevertheless, we could argue another possibility to explain this behaviour. Due to the specific interaction between the surfactant and the crosslinker, this could remain in the bilayers and, as a consequence, the crosslinking processes could not be efficient enough to produce a network. As a result, short uncrosslinked chains could remain partially in the lamellar structure, the BA units interacting with the surfactant polar heads, increasing its effective area, which could explain the decrease of both, $d_{0}$ and the polymer concentration in the isotropic phase.

Sample $71 \mathrm{E}_{\mathrm{P}}$ shows the same pattern as sample $71 \mathrm{E}_{0}$ indicating the coexistence of cubic and lamellar domains (see Fig. 3 and Fig. 5). For both samples, the long period of the lamellar domains is $d=2.72 \pm 0.01 \mathrm{~nm}$, and the cubic lattice parameter, calculated from the position of the first peak as $a_{C}=2 \pi 6^{1 / 2} / q$, is $a_{C}=6.95 \pm 0.05 \mathrm{~nm}$.

The sequence of ordered phases found here is qualitatively the same as in the AOT/water system [23]. Nevertheless, the presence of polymer produces a variation in the concentration at which the phases appear. The cubic phase appears in the binary system at $76 \mathrm{wt} \%$ but here this phase is produced at $71 \mathrm{wt} \%$, as expected in a system where there is a phase separation as was explained above.

\subsection{Hydrogel characterization}

In the following, the morphology of gels is determined and a model correlating this morphology with the structure of the synthesis medium is proposed. It has been done for samples $E_{P}$, because for the $E_{0}$ set, the amount of sample is not enough to allow for polymer 
characterization.

An important parameter related to the gel properties is the effective crosslinking. It is well known [39] that $T_{g}$ in polymer networks increases with the effective crosslink chain density. Fig. 7 shows that the samples studied here can be divided into two groups: the first one corresponds to the hydrogels obtained from samples with low AOT content ( $7 \mathrm{E}_{\mathrm{P}}$ and $14 \mathrm{E}_{\mathrm{P}}$ ), which have the same $\mathrm{T}_{\mathrm{g}}$ as the conventional hydrogel $(0 \mathrm{E})$, independently of the macroscopic phase from which they have been obtained. The second group is formed by hydrogels obtained with higher AOT volume fraction. These have lower $T_{g}$ values, close to the $T_{g}$ of the uncrosslinked polymer. Sample $21 \mathrm{E}_{\mathrm{P}}$ corresponds to the transition between both groups. The upper phase behaves as the samples with lower AOT concentration and the bottom phase behaves as the other group. Within each group, the $\mathrm{T}_{\mathrm{g}}$ does not vary significantly with the AOT concentration. Intuitively, one would say that the presence of high concentration (above a given threshold) of AOT in the reaction medium, induces lower crosslinking, since hydrogels synthesized with AOT above the threshold concentration have lower $T_{g}$, similar to that of the uncrosslinked polymer.
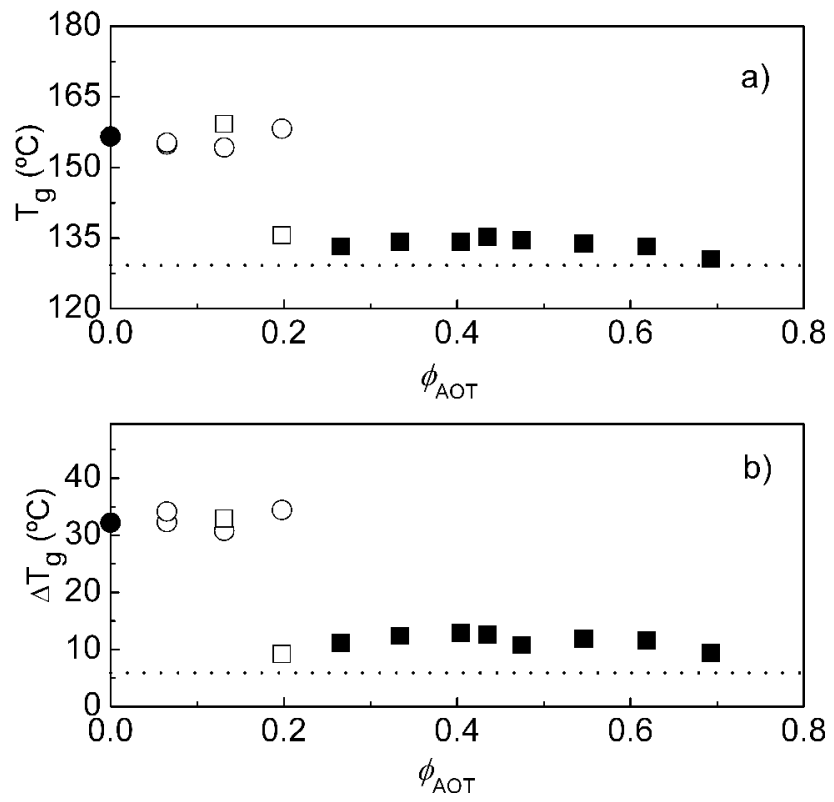

Fig. 7 a) Glass transition temperature, $T_{g}$, and b) width of $T_{g}\left(\Delta T_{g}\right)$ both depicted as a function of the global AOT volume fraction. Full symbols: samples with one macroscopic phase, open symbols: samples with several macroscopic phases. Squares correspond to the bottom phase and circles to the upper phase. The dotted line marks the value corresponding to a sample of uncrosslinked PDMAA with high molecular weight. For sample 7E the bottom phase has not been characterized because the amount of sample was insufficient. 
Not only $T_{g}$, but also the width of the glass transition $\left(\Delta T_{g}\right)$, calculated as the difference between the onset and endset temperatures, presents the same pattern. The characteristic length of glass transition $\xi\left(\mathrm{T}_{\mathrm{g}}\right)$, which corresponds to a length scale of the cooperative arrangement due to polymer segmental relaxation, depends [40] on $\left(\Delta \mathrm{T}_{\mathrm{g}}\right)^{-2 / 3}$, and it is known that $\xi\left(\mathrm{T}_{\mathrm{g}}\right)$ decreases with increasing crosslinking.

Therefore, the calorimetric results of $\Delta \mathrm{T}_{\mathrm{g}}$ also indicate a lower crosslinking of the hydrogels synthesized with higher AOT content. However the molecular architecture of the network and the crosslinking heterogeneity can also play an important role [41], so differences in the network structure cannot be disregarded, as it will be shown.

Another important parameter characteristic of the hydrogels is the degree of swelling. It depends on the crosslinking degree but also on the morphology, i.e. the size and the structure of pores. The gels obtained from the samples where there is macroscopic phase separation (phases: upper, U, central, C, and bottom, B) are mechanically consistent, with the exception of the hydrogel belonging to the sample $21 \mathrm{E}_{\mathrm{P}} \mathrm{B}$, and their swellings (see Table 3 ) are close to the conventional hydrogel $(0 \mathrm{E})$ swelling. This agrees with the $\mathrm{T}_{\mathrm{g}}$ results, and would indicate that these hydrogels have similar crosslinking density and morphology, as expected for hydrogels obtained in a medium with low surfactant content [24]. Hydrogels obtained with higher AOT content, are not mechanically consistent and it is not possible to obtain an accurate swelling value. 
Table 3 Swelling $(S)$ of consistent gels: sample $0 \mathrm{E}_{\mathrm{P}}$, and macroscopic phases (upper, $\mathrm{U}$, central, C, and bottom, B) of samples $7 \mathrm{E}_{\mathrm{P}}, 14 \mathrm{E}_{\mathrm{P}}$ and $21 \mathrm{E}_{\mathrm{P}}$. Microstructure characterization of the hydrogels from SEM micrographs (Pore size for sponge-like morphologies and fibril thickness corresponding to the fibrillar-like morphologies)

\begin{tabular}{lccc}
\hline Sample & $S$ & $\begin{array}{c}\text { Pore Size } \\
(\mu \mathrm{m})\end{array}$ & $\begin{array}{c}\text { Fibril Thickness } \\
(\mu \mathrm{m})\end{array}$ \\
\hline $0 \mathrm{E}_{\mathrm{P}}$ & $30.7 \pm 0.3$ & $50 \pm 40$ & - \\
$7 \mathrm{E}_{\mathrm{P}-\mathrm{U}}$ & $29.5 \pm 0.3$ & $3 \pm 4$ & - \\
$7 \mathrm{E}_{\mathrm{P}-\mathrm{C}}$ & $34 \pm 1$ & $20 \pm 20$ & - \\
$14 \mathrm{E}_{\mathrm{P}-\mathrm{U}}$ & $30.9 \pm 0.2$ & $100 \pm 50$ & $0.59 \pm 0.09$ \\
$14 \mathrm{E}_{\mathrm{P}-\mathrm{B}}$ & $26 \pm 2$ & $120 \pm 40$ & $1.0 \pm 0.2$ \\
$21 \mathrm{E}_{\mathrm{P}-\mathrm{U}}$ & $23 \pm 1$ & $130 \pm 70$ & $0.4 \pm 0.1$ \\
$21 \mathrm{E}_{\mathrm{P}-\mathrm{B}}$ & - & - & $1.0 \pm 0.2$ \\
$29 \mathrm{E}_{\mathrm{P}}$ & - & - & $0.9 \pm 0.2$ \\
$36 \mathrm{E}_{\mathrm{P}}$ & - & - & $0.8 \pm 0.2$ \\
$43 \mathrm{E}_{\mathrm{P}}$ & - & - & $0.5 \pm 0.1$ \\
$46 \mathrm{E}_{\mathrm{P}}$ & - & - & $0.29 \pm 0.07$ \\
$57 \mathrm{E}_{\mathrm{P}}$ & - & - & $0.6 \pm 0.2$ \\
$64 \mathrm{E}_{\mathrm{P}}$ & - & - & $0.8 \pm 0.1$ \\
$71 \mathrm{E}_{\mathrm{P}}$ & - & - & $0.8 \pm 0.2$ \\
\hline & & &
\end{tabular}

Fig. 8 illustrates the morphology found in the hydrogels. A comparison of these micrographs shows three groups of samples. (i) Hydrogels generated from the sample with the lowest AOT content $\left(7 \mathrm{E}_{\mathrm{P}}-\mathrm{U}, 7 \mathrm{E}_{\mathrm{P}}-\mathrm{C}\right)$ have a sponge-like morphology similar to the conventional hydrogel $\left(0 \mathrm{E}_{\mathrm{P}}\right)$. (ii) Hydrogels coming from laminar monophasic samples $\left(29 \mathrm{E}_{\mathrm{P}}-71 \mathrm{E}_{\mathrm{P}}\right)$ have a fibrillarlike morphology. (iii) In the intermediate cases the hydrogels present a hierarchical structure formed by closed pores with a secondary fibrillar-like structure filling the pores. For samples $14 \mathrm{E}_{\mathrm{P}}-\mathrm{U}$ and $14 \mathrm{E}_{\mathrm{P}}-\mathrm{B}$, the amount of the fibrillar structure is not very high, while in sample $21 \mathrm{E}_{\mathrm{P}}-\mathrm{U}$, the pores are completely filled and the fibrils are highly organized inside the pores (see Fig. 9). 

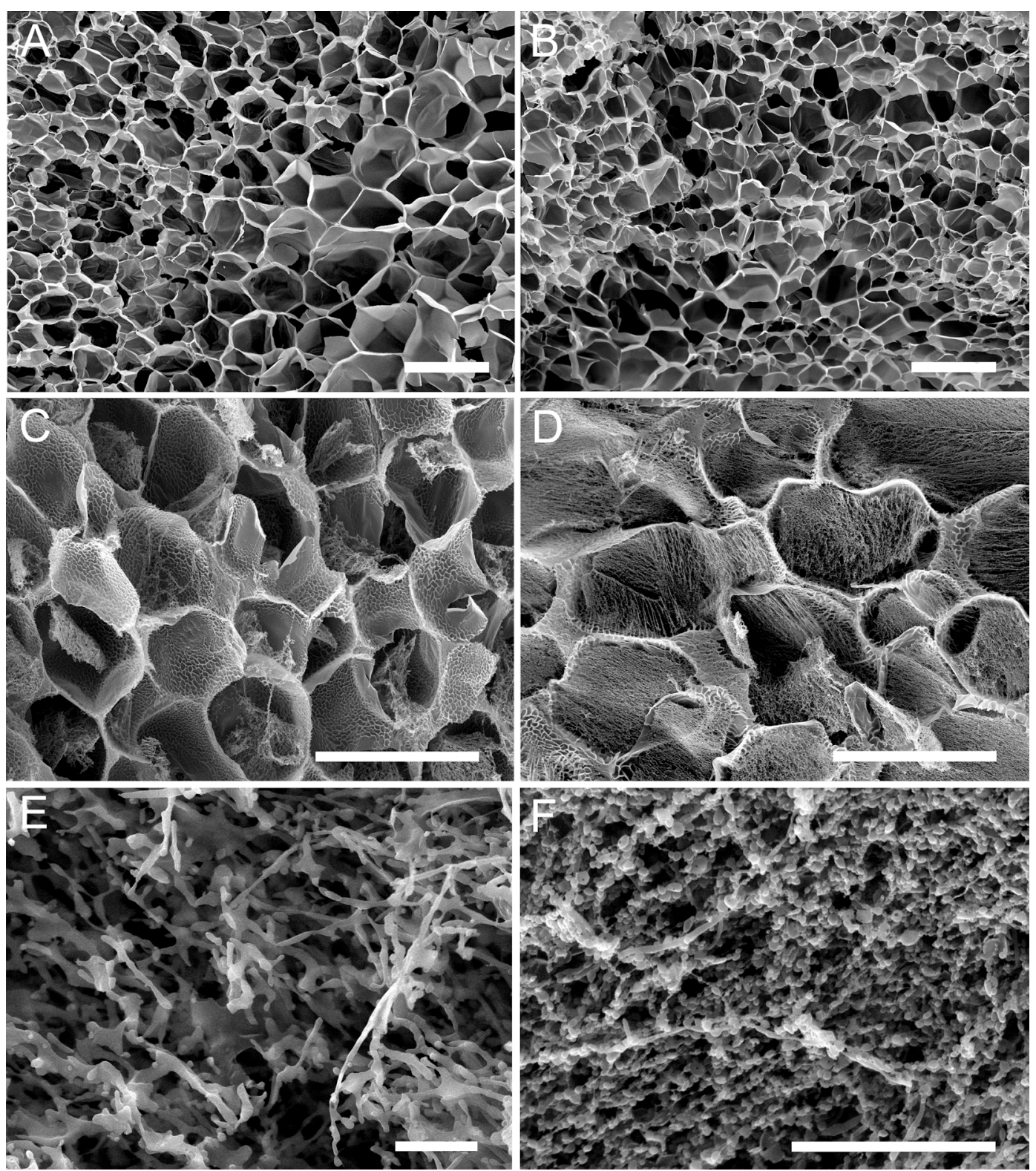

Fig. 8 SEM micrographs of selected samples. Bar $\left.\left.\left.=200 \mu \mathrm{m}: \mathrm{A}) 0 \mathrm{E}_{\mathrm{P}}, \mathrm{B}\right) 7 \mathrm{E}_{\mathrm{P}}-\mathrm{C}, \mathrm{C}\right) 14 \mathrm{E}_{\mathrm{P}}-\mathrm{U}, \mathrm{D}\right)$ $21 \mathrm{E}_{\mathrm{P}}-\mathrm{U} . \mathrm{Bar}=10 \mu \mathrm{m}:$ E) $29 \mathrm{E}_{\mathrm{P}}$ and F) $46 \mathrm{E}_{\mathrm{P}}$.

These morphologies of the hydrogels can be correlated with the properties determined above for their characterization. It can be observed that the hydrogels with sponge-like structure are mechanically consistent and present a higher $T_{g}$ while the fibrillar ones are not mechanically consistent and present lower $\mathrm{T}_{\mathrm{g}}$ values. Regarding the swelling results, hydrogel $7 \mathrm{E}_{\mathrm{P}}-\mathrm{U}$ has a higher swelling than $7 \mathrm{E}_{\mathrm{P}}-\mathrm{C}$, as expected from the pore size estimation. Nevertheless, for the hydrogels $14 \mathrm{E}_{\mathrm{P}}-\mathrm{U}, 14 \mathrm{E}_{\mathrm{P}}-\mathrm{B}$ and $21 \mathrm{E}_{\mathrm{P}}-\mathrm{U}$, the higher pore size does not mean a higher swelling degree, because of the secondary fibrillar structure filling the pores (Fig. 9). 


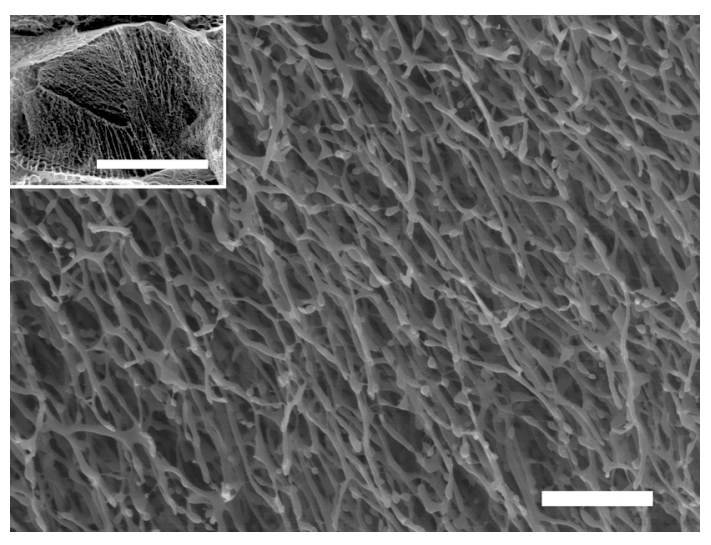

Fig. 9 SEM micrographs of sample $21 \mathrm{E}_{\mathrm{P}}-\mathrm{U}$ showing the secondary structure filling the pores (bar $=10 \mu \mathrm{m})$. The insert corresponds to a representative pore $(\mathrm{bar}=100 \mu \mathrm{m})$.

To explain the morphology of the samples studied here, we should take into account that, as the polymerization and crosslinking evolves, the local AOT/water partitions into two phases, one containing the polymer and other (mesophase) excluding the polymer. The interplay of the growth kinetics of the ordered domains and the monomer conversion kinetics sets the size of the pores in the final crosslinked gel. Its architecture is the result of the demixing [19], and the gel morphology depends on the step in which gelation takes place. Therefore, the following model could be proposed:

a) In hydrogels generated from the isotropic samples with the lowest AOT content, the lamellar domains are formed when the polymerization is almost concluded. For this reason, the morphology is similar to that observed for conventional gels, as it has been previously reported [24]. Furthermore, the differences of their pore size are due to the different concentration of the comonomers as a consequence of the macroscopic phase separation process. The results obtained here would indicate that the upper phase has a higher polymer concentration, which agree with a previous study [16].

b) In hydrogels generated from samples with lamellar order (i.e. samples with highest AOT content) the polymer segregates from the already existing lamellar domains. Therefore, the coarsening of the polymer structure is hindered and stopped in the first steps and as a consequence, open pores with fibrillar structure are formed, rendering hidrogels which are not mechanically consistent.

c) The other samples present an intermediate behaviour. At the first steps of polymerization the lamellar domains does not exist and sponge-like structures are formed. When the 
polymerization progresses, lamellar domains are formed and there is monomer enough to continue the polymerization in-between the ordered domains, giving rise to the secondary fibrillar structure. In fact, it is noteworthy that the fibrils grow inside the pores with preferred orientations. The hydrogel from sample $14 \mathrm{E}_{\mathrm{P}}-\mathrm{B}$ having initial lamellar order exhibits a morphology similar to that of hydrogels generated from the isotropic phases. This can be understood after considering that it comes from a sample in which a phase separation process has taken place before polymerization. When this occurs, the splitting between both phases could not be complete. In the present case, the bottom lamellar phase seems to contain sufficient amount of isotropic domains to generate sponge-like morphologies.

Table 3 shows that for the macroscopic monophasic samples, the thickness of the fibrils presents a minimum for an AOT concentration around $46 \mathrm{wt} \%$. In order to understand this behaviour, it should be taken into account that there is a considerable change in some physical properties of the AOT/water system around this concentration: there is a change in the sign of the birefringence [29], the conductivity reaches a maximum [31], no bulk-like water is detected [42], there is a broadening of the ${ }^{1} \mathrm{H}-\mathrm{NMR}$ water resonance [43]. Additionally, the viscosity shows a minimum at this concentration, which has been explained [44] as a minimum in the coulombic interactions, resulting in weaker interactions, a decrease of connectivity among microdomains, and more structural defects. As was explained above, the demixing and therefore the rheology has a strong influence on the final morphology. In this case, the significant lower viscosity, as a consequence of the smaller connectivity of the liquid crystalline domains, seems to be a driving force for the formation of thinner fibrillar structure. The morphology of gel is directed by the nucleation and growth processes which are conditioned by the liquid crystalline template, and in this case, as a result of the smaller connectivity, it can be expected more nucleation points giving rise to a thinner structure. The fibril thickness $(0.3-1 \mu \mathrm{m})$ has similar scale to those of some natural structures (e.g. nerves). On the other hand the thickness can be modulated by the physical properties of the lyotropic medium. Therefore, this method could be adequate to produce scaffold for tissue engineering.

\section{Conclusions}

The initial composition, the type of ordered structure, and the polymer segregation process all influence the characteristics of polydimethylacrylamide hydrogels obtained in the $\mathrm{AOT} / \mathrm{H}_{2} \mathrm{O}$ system. 
Hydrogels formed by fibrillar networks are obtained when the polymerization is performed in a lamellar system because the coarsening of the polymer structure is hindered and stopped in the first steps. The fibril thickness seems to be correlated with the rheology of the lamellar mesophase.

Hierarchical structures formed by closed pores with a secondary fibrillar structure filling the pores are obtained from samples with an initial isotropic structure that becomes lamellar with polymerization. The sponge-like structures are formed at the first steps of polymerization. When the polymerization progresses lamellar domains are formed and fibrils start to grow inside the pores, with preferred orientations, giving rise to the secondary fibrillar structure. This study may open a new way to obtain materials with an adequate morpholgy to produce scaffolds.

\section{Acknowledgements}

This work received financial support from MICINN (Spain), under grant CTQ2010-16414, and from DGUI (Comunidad de Madrid), under R\&D Program MODELICO-CM/S2009ESP1691. We are indebted for beam time in the line BM16 of the ESRF (Grenoble). Prof. Arturo Horta is gratefully acknowledged for helpful discussion and advice. 


\section{References}

1. Singh A, Sarkar DJ, Singh AK, Parsad R, Kumar A, Parmar BS. Studies on novel nanosuperabsorbent composites: Swelling behavior in different environments and effect on water absorption and retention properties of sandy loam soil and soil-less medium. J. Appl. Polym. Sci. 2011; 120: 1448-1458.

2. Zohuriaan-Mehr MJ, Omidian H, Doroudiani S, Kabiri K. Advances in non-hygienic applications of superabsorbent hydrogel materials. J. Mater. Sci. 2010; 45: 5711-5735.

3. Simhadri JJ, Stretz HA, Oyanader M, Arce PE. Role of nanocomposite hydrogel morphology in the electrophoretic separation of biomolecules: A review. Ind. Eng. Chem. Res. 2010; 49: 11866-11877.

4. Kopeck J. Hydrogels: From soft contact lenses and implants to self-assembled nanomaterials. J. Polym. Sci. Part A Polym. Chem. 2009; 47: 5929-5946.

5. Hoffman AS. Hydrogels for biomedical applications. Adv. Drug Deliver. Rev. 2002; 43: 312.

6. Peppas NA, Bures P, Leobandung W, Ichikawa H. Hydrogels in pharmaceutical formulations. Eur. J. Pharm. Biopharm. 2000;20: 27-46.

7. Annabi N, Nichol JW, Zhong X, Ji C, Koshy S, Khademhosseini A, Dehghani F. Controlling the porosity and microarchitecture of hydrogels for tissue engineering. Tissue Eng. B 2010; 16: 371-383.

8. da Silva R, de Oliveira MG. Effect of the cross-linking degree on the morphology of poly(NIPAAm-co-AAc) hydrogels. Polymer 2007; 48: 4114-4122.

9. Collins MN, Birkinshaw C. Morphology of crosslinked hyaluronic acid porous hydrogels. J. Appl. Polym. Sci. 2011; 120: 1040-1049.

10. Zawko SA, Schmidt CE. Crystal templating dendritic pore networks and fibrillar microstructure into hydrogels. Acta Biomater. 2010; 6: 2415-2421.

11. Alvarez-Perez MA, Guarino V, Cirillo V, Ambrosio L. Influence of gelatin cues in PCL electrospun membranes on nerve outgrowth. Biomacromolecules 2010; 11:2238-2246.

12. Brizard AM, Esch JH. Self-assembly approaches for the construction of cell architecture mimics. Soft Matter 2009; 5: 1320-1327.

13. Laversanne R. Polymerization of acrylamide in lamellar, hexagonal, and cubic lyotropic phases. Macromolecules, 1992; 25: 489-491.

14. Texter J. Templating hydrogels. Colloid Polym. Sci. 2009; 287: 313-321.

15. Hentze HP, Kaler EW. Morphosynthesis of nanostructured polymer gels by polymerization within reverse hexagonal mesophases. Chem. Mater. 2003; 15: 708-713.

16. Pacios IE, Horta A, Renamayor CS. Macroporous gels of poly( $N, N$-dimethylacrylamide) obtained in the lamellar system AOT/water. Macromolecules 2004; 37: 4643-4650. 
17. Hentze HP, Göltner CG, Antonietti M. Synthesis of organic polymer gels in microemulsions and lyotropic mesophases. Ber. Bunsenges. Phys. Chem. 1997; 101: 16991702.

18. Hentze HP, Kaler EW. Polymerization of and within self-organized media. Curr. Opin. Colloid Interface Sci. 2003; 8: 164-178.

19. Hentze HP, Antonietti M. Template synthesis of porous organic polymers. Curr. Opin. Solid State Mater. Sci. 2001; 5: 343-353.

20. Antonietti M, Caruso RA, Hentze HP, Göltner C. Hydrophilic gels with new superstructures and their hybrids by nanocasting technologies. Macromol. Symp. 2000; 152: $163-172$.

21. de Pierro MA, Guymon CA. Photoinitiation and monomer segregation behavior in polymerization of lyotropic liquid crystalline systems. Macromolecules 2006; 39: 617-626.

22. Lester CL, Smith SM, Jarret WL, Guymon CA. Effects of monomer organization on the photopolymerization kinetics of acrylamide in lyotropic liquid crystalline phases. Langmuir 2003; 19: 9466-9472.

23. Pacios IE, Renamayor CS. Induced phase transitions by cross-linking polymerization of $\mathrm{N}, \mathrm{N}$-dimethylacrylamide within isotropic AOT/water phases.J. Phys. Chem. B 2009; 113: 16494-16500.

24. Renamayor CS, Pacios IE. Porous structures controlled by segregation of ordered mesophases in poly $(N, N$-dimethylacrylamide) hydrogels polymerized from an isotropic AOT/water medium. Soft Matter 2010; 6: 2013-2020.

25. Rajaram CV, Hudson SD, Chien LC. Morphology of polymer-stabilized liquid crystals. Chem. Mater. 1995; 7: 2300-2308.

26. Lapeña AM, Glotzer SC, Langer SA, Liu AJ. Effect of ordering on spinodal decomposition of liquid-crystal/polymer mixtures. Phys. Rev. E 1999; 60: R29-R32

27. Abramoff MD, Magelhaes PJ, Ram SJ. Image processing with ImageJ. Biophoton Int. 2004; 11: 36-42.

28. Rogers J, Winsor PA. Change in the optic sign of the lamellar phase $(G)$ in the aerosol OT/water system with composition or temperature. J. Colloid Interface Sci. 1969; 30: 247257.

29. Rogers J, Winsor PA. Optically positive isotropic and negative lamellar liquid crystalline solutions. Nature 1967; 216: 477-479.

30. Boltenhagen P, Lavrentovich O, Kleman M. Oily streaks and focal conic domains in $\mathrm{L}_{\alpha}$ lyotropic liquid crystals. J. Phys. II France 1991; 1: 1233-1252.

31. Fontell K. J. Colloid Interface Sci. The structure of the lamellar liquid crystalline phase in the aerosol OT-water system. 1973; 44: 318-329. 
32. Ficheux MF, Bellocq AM, Nallet F. Experimental-study of a lyotropic lamellar phase swollen with polymer-solutions. J. Phys. II France 1995; 5: 823-834.

33. Puvvada S, Qadri SB, Naciri J, Ratna BR. Effect of acrylamide and dodecane on the phase behavior of monoolein. Langmuir 1994; 10: 2972-2976.

34. Seguin C, Eastoe J, Heenan RK, Grillo I. SANS studies of the effects of surfactant head group on aggregation properties in water/glycol and pure glycol systems. J. Colloid Interface Sci. 2007; 315: 714-720.

35. Tirumala VR, Caneba GT, Mancini DC, Wang HH. Microfabrication by X-ray-induced polymerization above the lower critical solution temperature. J. Appl. Polym. Sci. 2006; 102: 429-435.

36. Demé B, Dubois M, Zemb T, Cabane B. J. Phys. Chem. Effect of carbohydrates on the swelling of a lyotropic lamellar phase. 1996; 100: 3828-3838.

37. Pacios IE, Renamayor CS, Horta A, Lindman B, Thuresson K. In situ polymerization of $N, N$-dimethylacrylamide in aerosol OT-water: Modified lamellar structure and multiphase separation. Macromolecules 2002; 35: 7553-7560.

38 Nallet F, Laversanne R, Roux D. Modeling X-ray or neutron-scattering spectra of lyotropic lamellar phases-interplay between form and structure factors. J. Phys II 1993; 3: 487-502.

39. DiMarzio EA. On second-order transition of rubber. J. Res. Natl. Bur. Stand. A Phys. Chem. 1964; 68: 611-617.

40. Donth E. Characteristic length of the glass transition. J. Polym. Sci. Part B 1996; 26: 2881-2892.

41. Sasaki T, Uchida T, Sakurai K. Effect of crosslink on the characteristic length of glass transition of network polymers. J. Polym. Sci. Part B 2006; 44: 1958-1966.

42. Casillas N, Puig JE, Olayo R, Hart TJ, Franses EI. State of water and surfactant in lyotropic liquid crystals. Langmuir 1989; 5: 384-389.

43. Franses EI, Hart TJ. Phase-behavior and molecular-motion of aerosol OT in liquidcrystalline phases with water. J. Colloid Interface Sci. 1983; 94: 1-13.

44. Robles-Vásquez O, Corona-Galván S, Soltero JFA, Puig JE, Tripodi SB, Vallés E, Manero O. Rheology of lyotropic liquid-crystals of aerosol OT .2. High-concentration regime. J. Colloid Interface Sci. 1993; 160: 65-75. 


\section{FIGURE CAPTIONS}

Fig. 1 Micrographs with crossed polarizers at room temperature before polymerization corresponding to samples: A) $29 \mathrm{E}_{\mathrm{P}}$, B) $46 \mathrm{E}_{\mathrm{P}}$ and C) $57 \mathrm{E}_{\mathrm{P}}($ bar $=100 \mu \mathrm{m})$.

Fig. 2 X-ray induced polymerization of sample $46 \mathrm{E}_{0}$. Selected SAXS diffractograms for cumulative irradiation times in the range $0.5-60 \mathrm{~s}$, starting at the bottom of the graph. SAXS profiles have been progressively shifted upwards for better visualization. Intensity in arbitrary units.

Fig. 3 X-ray induced polymerization of sample $71 \mathrm{E}_{0}$. Selected SAXS diffractograms for cumulative irradiation times in the range $0.5-60 \mathrm{~s}$, starting at the bottom of the graph. SAXS profiles have been progressively shifted upwards for better visualization. The insert is an amplification of the region $2.25-2.35 \mathrm{~nm}^{-1}$ showing the appearance of a shoulder in the first order diffraction of the lamellar phase. Intensity in arbitrary units.

Fig. 4 Micrographs corresponding to the upper phase of sample $14 \mathrm{E}_{\mathrm{P}}$, without (left) and with

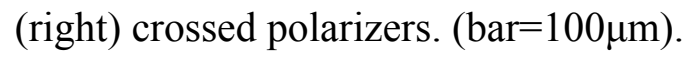

Fig. 5 SAXS diffractograms of macroscopic monophasic samples corresponding to samples polymerized with AIBN $\left(E_{P}\right)$. SAXS profiles have been progressively shifted upwards for better visualization. Intensity in logarithmic scale (arbitrary units).

Fig. 6 Inverse of the long period $(1 / d)$ after polymerization as a function of global AOT volume fraction, $\phi_{\mathrm{AOT}}$, Samples $\mathrm{E}_{\mathrm{P}}$ : squares and dotted line; samples $\mathrm{E}_{0}$ : diamonds and solid line. 
Fig. 7 a) Glass transition temperature, $T_{g}$, and b) width of $T_{g}\left(\Delta T_{g}\right)$ both depicted as a function of the global AOT volume fraction. Full symbols: samples with one macroscopic phase, open symbols: samples with several macroscopic phases. Squares correspond to the bottom phase and circles to the upper phase. The dotted line marks the value corresponding to a sample of uncrosslinked PDMAA with high molecular weight. For sample 7E the bottom phase has not been characterized because the amount of sample was insufficient.

Fig. 8 SEM micrographs of selected samples. Bar $\left.\left.\left.=200 \mu \mathrm{m}: \mathrm{A}) 0 \mathrm{E}_{\mathrm{P}}, \mathrm{B}\right) 7 \mathrm{E}_{\mathrm{P}}-\mathrm{C}, \mathrm{C}\right) 14 \mathrm{E}_{\mathrm{P}}-\mathrm{U}, \mathrm{D}\right)$ $21 \mathrm{E}_{\mathrm{P}}-\mathrm{U}$. Bar $=10 \mu \mathrm{m}:$ E) $29 \mathrm{E}_{\mathrm{P}}$ and F) $46 \mathrm{E}_{\mathrm{P}}$.

Fig. 9 SEM micrographs of sample $21 \mathrm{E}_{\mathrm{P}}-\mathrm{U}$ showing the secondary structure filling the pores (bar $=10 \mu \mathrm{m})$. The insert corresponds to a representative pore $(\mathrm{bar}=100 \mu \mathrm{m})$. 


\section{TABLES}

Table 1 AOT concentration of the samples

\begin{tabular}{llll}
\hline Sample & $\begin{array}{l}\text { AOT } \\
(\mathrm{wt} \%)\end{array}$ & Sample & $\begin{array}{l}\text { AOT } \\
(\mathrm{wt} \%)\end{array}$ \\
\hline $0 \mathrm{E}$ & 0 & $43 \mathrm{E}$ & 42.9 \\
$7 \mathrm{E}$ & 7.1 & $46 \mathrm{E}$ & 45.9 \\
$14 \mathrm{E}$ & 14.3 & $50 \mathrm{E}$ & 49.9 \\
$21 \mathrm{E}$ & 21.4 & $57 \mathrm{E}$ & 57.1 \\
$29 \mathrm{E}$ & 28.6 & $64 \mathrm{E}$ & 64.2 \\
$36 \mathrm{E}$ & 35.7 & $71 \mathrm{E}$ & 71.4 \\
\hline
\end{tabular}

Table 2 Lamellar spacing corresponding to the macroscopic phases (upper and bottom) of the samples with lower AOT content after polymerization.

\begin{tabular}{lccccc}
\hline \multirow{2}{*}{ Sample } & \multicolumn{2}{c}{ AIBN } & & \multicolumn{2}{c}{ X- ray } \\
\cline { 2 - 3 } \cline { 6 - 6 } & Upper & Bottom & & Upper & Bottom \\
& $d(\mathrm{~nm})$ & $d(\mathrm{~nm})$ & & $d(\mathrm{~nm})$ & $d(\mathrm{~nm})$ \\
\hline $7 \mathrm{E}$ & $15 \pm 1 \quad 13 \pm 1^{\mathrm{a}}$ & $-^{\mathrm{b}}$ & & - & $12.6 \pm 0.1$ \\
$14 \mathrm{E}$ & $8.8 \pm 0.2$ & $8.63 \pm 0.02$ & & - & $11.0 \pm 0.1$ \\
$21 \mathrm{E}$ & $7.36 \pm 0.06$ & $7.05 \pm 0.05$ & & $8.7 \pm 0.1$ & \\
\hline
\end{tabular}

${ }^{\mathrm{b}}$ The amount of sample in this region was not enough to allow the SAXS characterization. 
Table 3 Swelling $(S)$ of consistent gels: sample $0 \mathrm{E}_{\mathrm{P}}$, and macroscopic phases (upper, U, central, C, and bottom, B) of samples $7 \mathrm{E}_{\mathrm{P}}, 14 \mathrm{E}_{\mathrm{P}}$ and $21 \mathrm{E}_{\mathrm{P}}$. Microstructure characterization of the hydrogels from SEM micrographs (Pore size for sponge-like morphologies and fibril thickness corresponding to the fibrillar-like morphologies)

\begin{tabular}{lccc}
\hline Sample & $S$ & $\begin{array}{c}\text { Pore Size } \\
(\mu \mathrm{m})\end{array}$ & $\begin{array}{c}\text { Fibril Thickness } \\
(\mu \mathrm{m})\end{array}$ \\
\hline $0 \mathrm{E}_{\mathrm{P}}$ & $30.7 \pm 0.3$ & $50 \pm 40$ & - \\
$7 \mathrm{E}_{\mathrm{P}-\mathrm{U}}$ & $29.5 \pm 0.3$ & $3 \pm 4$ & - \\
$7 \mathrm{E}_{\mathrm{P}-\mathrm{C}}$ & $34 \pm 1$ & $20 \pm 20$ & - \\
$14 \mathrm{E}_{\mathrm{P}-\mathrm{U}}$ & $30.9 \pm 0.2$ & $100 \pm 50$ & $0.59 \pm 0.09$ \\
$14 \mathrm{E}_{\mathrm{P}}-\mathrm{B}$ & $26 \pm 2$ & $120 \pm 40$ & $1.0 \pm 0.2$ \\
$21 \mathrm{E}_{\mathrm{P}-\mathrm{U}}$ & $23 \pm 1$ & $130 \pm 70$ & $0.4 \pm 0.1$ \\
$21 \mathrm{E}_{\mathrm{P}-\mathrm{B}}$ & - & - & $1.0 \pm 0.2$ \\
$29 \mathrm{E}_{\mathrm{P}}$ & - & - & $0.9 \pm 0.2$ \\
$36 \mathrm{E}_{\mathrm{P}}$ & - & - & $0.8 \pm 0.2$ \\
$43 \mathrm{E}_{\mathrm{P}}$ & - & - & $0.5 \pm 0.1$ \\
$46 \mathrm{E}_{\mathrm{P}}$ & - & - & $0.29 \pm 0.07$ \\
$57 \mathrm{E}_{\mathrm{P}}$ & - & - & $0.6 \pm 0.2$ \\
$64 \mathrm{E}_{\mathrm{P}}$ & - & - & $0.8 \pm 0.1$ \\
$71 \mathrm{E}_{\mathrm{P}}$ & - & - & $0.8 \pm 0.2$ \\
\hline & & &
\end{tabular}

